\title{
Is the intravenous giving set a reliable alternative to the spinal manometer in measuring cerebrospinal fluid opening pressure?
}

\author{
T A Mogambery, ${ }^{1} \mathrm{MB}$ ChB, FCP; A Moodley, ${ }^{2} \mathrm{MB}$ ChB, FCP Neurol, PhD; C Connolly, ${ }^{3} \mathrm{PhD}$ \\ ${ }^{1}$ Department of Medicine, Edendale Hospital, Pietermaritzburg, and Nelson R Mandela School of Medicine, University of KwaZulu-Natal, \\ Durban, South Africa \\ ${ }^{2}$ Department of Neurology, Universitas Hospital, University of the Free State, Bloemfontein, South Africa \\ ${ }^{3}$ Department of Biostatistics, Nelson R Mandela School of Medicine, University of KwaZulu-Natal, Durban, South Africa
}

Corresponding author: A Moodley (moodleyaa@ufs.ac.za)

\begin{abstract}
Background. Measurement of the cerebrospinal fluid (CSF) opening pressure (OP) during lumbar puncture (LP) should be routine practice. In resource-limited centres, spinal manometers are seldom available and alternative procedures to measure CSF OP are undertaken. Objectives. To investigate whether the intravenous giving set (IVGS) with a measuring tape is a reliable alternative to the spinal manometer. Methods. One hundred patients requiring CSF examination by LP were consecutively recruited in the Department of Medicine at Edendale Hospital, Pietermaritzburg, South Africa. A three-way stopcock was attached to the end of a 22G spinal needle and the IVGS and spinal manometer were attached to the other two openings of the stopcock. CSF OP was consecutively recorded between the two techniques with 50 patients in each group.

Results. The mean (standard deviation (SD)) CSF OP of the 100 patients was 22.7 (10.0) cm CSF measured with the manometer v. 16.2 (9.3) $\mathrm{cm}$ CSF measured with the IVGS $(\mathrm{p}<0.001)$. Subgroup analysis showed similarly significant findings of consistently lower CSF OP with the IVGS, regardless of whether the IVGS reading was done first or second. The manometer detected 34 cases of elevated CSF OP of $>25 \mathrm{~cm}$ CSF, but the IVGS detected 11 cases only ( $p<0.001$, McNemar's $\chi^{2}$ test). Similar differences were noted for the subgroups of manometer first and IVGS first. Despite this, linear correlation showed very good correlation $(r=0.78)$ and a $75 \%$ agreement between the two techniques. The relationship between the manometer reading and the IVGS reading was $\mathrm{M}=0.85 \mathrm{~V}+8.9$ in $\mathrm{cm}$ CSF, where $\mathrm{M}$ was the manometer reading and $\mathrm{V}$ the IVGS reading.

Conclusions. The IVGS consistently underestimated the CSF OP against the tried-and-tested spinal manometer, which should be the preferred method of measuring CSF OP. Based on the equation that describes the relationship between the spinal manometer and IVGS reading, the upper limit of normal CSF OP of $25 \mathrm{~cm}$ CSF on the manometer is equivalent to $19 \mathrm{~cm}$ CSF on the IVGS.
\end{abstract}

S Afr Med J 2018;108(10):865-869. DOI:10.7196/SAMJ.2018.v108i10.13176

Cerebrospinal fluid (CSF) examination by lumbar puncture (LP) is one of the most common procedures undertaken for diagnosis and therapy in an outpatient and inpatient setting in medical patients. CSF opening pressure $(\mathrm{OP})$ readings are a vital component of LPs and results often play an important role in guiding both diagnosis and management of patients with conditions such as cryptococcal meningitis, tuberculous meningitis, pseudotumour cerebri and normal-pressure hydrocephalus. ${ }^{[1-5]}$ In fact, CSF OP measurement should be standard procedure for every LP performed, and its omission points to an incomplete procedure.

Elevated intracranial pressure, defined as a CSF OP of $>25 \mathrm{~cm}$ CSF, is associated with increased mortality. Routine measurement and aggressive management of raised intracranial pressure are therefore vital. ${ }^{[6,7]}$

The standard method of measuring CSF OP is with a spinal manometer, but in many resource-limited centres manometers are not readily available. The cost of an intravenous giving set (IVGS) is ZAR1.77 and that of a disposable spinal manometer is ZAR70.00 (Adcock Ingram, January 2018). The substantial price difference and the difficulty in procuring spinal manometers, which are considered non-stock items, result in unavailability of spinal manometers in peripheral hospitals.
A single Tanzanian study has validated the use of the IVGS as an alternative to the spinal manometer. Good correlation was found between the spinal manometer and the IVGS in 35 CSF OP readings $\left(r^{2}=0.96\right.$ on the Bland-Altman plot). ${ }^{[8]}$ In this study manometer measurements were taken first in all cases followed by IVGS measurements, with loss of CSF at instrument changeover between manometer and IVGS not accounted for. The objective of the study was to demonstrate the benefit of lowering CSF pressure in cryptococcal meningitis. The comparison between manometer and IVGS OP was a secondary goal. The median pressure obtained with the manometer was $32 \mathrm{~cm}$ CSF (interquartile range (IQR) 24 - 37) and with the IVGS $30 \mathrm{~cm}$ CSF (IQR 23 - 35). Ninety-one percent of the patients had an elevated CSF pressure, which in most cases was still elevated but lower 14 days later. The authors recommended using the IVGS tubing as an alternative to the spinal manometer in resource-limited centres without any corrective factor between the two methods. With $91 \%$ of their patients having considerably elevated pressure, a wider spectrum of pressure measurement was not compared, especially that within the normal range.

Several alternative ways to measure CSF OP have been explored in order to find a simpler, cheaper and more accessible method for resource-limited settings where standard manometers are not 
readily available. Assessing OP by measuring capillary readings of CSF and CSF flow rate have been documented as successful methods, but are complicated and require user training to ensure accuracy of results. ${ }^{[9-11]}$ Pfeiffer et al. ${ }^{[11]}$ measured the CSF flow rate in a capillary tube between two target points and were able to show a qualitative relationship between elevated CSF pressure and the CSF flow rate. In an in vitro study, Ellis et al. ${ }^{[9]}$ demonstrated a relationship between CSF flow rate from the needle hub and CSF pressure. The CSF flow through a spinal needle was described by the equation $Q=$ $P / K V$, where $Q$ was the CSF flow rate in drops/minute, $P$ was the CSF pressure in $\mathrm{cm}$ CSF, $V$ was the relative viscosity of CSF to normal saline and $K$ was the constant for each sized needle. By counting the rate of drops from the spinal needle hub, a fairly accurate estimation of CSF pressure was made. Boyles et al. ${ }^{[10]}$ extrapolated this work to patients with cryptococcal meningitis and elevated CSF pressure in their Western Cape cohort. The limitation of this method encountered in their study was the difficulty in counting CSF drops when there was a continuous flow rather than separate drops, as is common when the intracranial pressure is severely elevated.

We attempted to address these shortcomings in the present study by using both the spinal manometer and the IVGS. We postulated more accurate readings by measuring the CSF OP consecutively either with the manometer first or with the IVGS first. In addition, CSF loss and especially pressure loss were avoided by using a threeway stopcock. Rather than a qualitative assessment of CSF pressure, we opted to use the IVGS and a measuring tape, which are readily available and already used by many units in resource-limited centres.

\section{Objectives}

The primary goal was to determine whether the larger-bore IVGS ( $3 \mathrm{~mm}$ ) was as accurate as the narrow-bore spinal manometer $(1 \mathrm{~mm})$ in measuring CSF OP. The secondary goal was to identify a mathematical model that best correlates the relationship between the measurements with the IVGS and spinal manometer if disparities were found, to provide a corrective factor for the IVGS if its use is unavoidable.

\section{Methods \\ Study design}

This was a prospective study where CSF OP was measured using both standard spinal manometers and IVGSs in 100 patients in an inpatient and outpatient setting.

\section{Study population}

A sample of 100 adult patients who required CSF examination by LP as part of their clinical work-up or management were identified at Edendale Hospital in Pietermaritzburg, South Africa (SA), between May 2017 and August 2017. Edendale Hospital is a 900-bed regional and district hospital in KwaZuluNatal Province that serves a population of $\sim 860000$ people in periurban and rural settings. It is the fourth-largest hospital in the country in terms of available beds.

\section{Ethical considerations}

Written and verbal informed consent to participate in the study were obtained from each patient or their next of kin (when the patient could not provide informed consent). The study was approved by the University of KwaZulu-Natal Biomedical Research Ethics Committee (ref. no. KZ 2017 RPI 223).

\section{Reference method of measuring CSF OP}

A single operator performed all LPs to exclude user variability. A 22G (colourcoded black) spinal needle of $90 \mathrm{~mm}$ length was used for all LPs. With the patient lying in the left lateral position, CSF OP was measured using a standard narrow-gauge $(1 \mathrm{~mm})$ manometer either before or after using a 20-dropper IVGS (3 mm). Raised intracranial pressure was defined as an $\mathrm{OP}$ $>25 \mathrm{~cm} \mathrm{CSF}^{[6]}$

\section{Investigational measurements of CSF OP}

A three-way stopcock was attached to the LP needle. Of the two remaining ports on the three-way stopcock, one was attached to the standard spinal manometer and the other to the IVGS. This allowed easy and quick transfer of CSF flow to the manometer

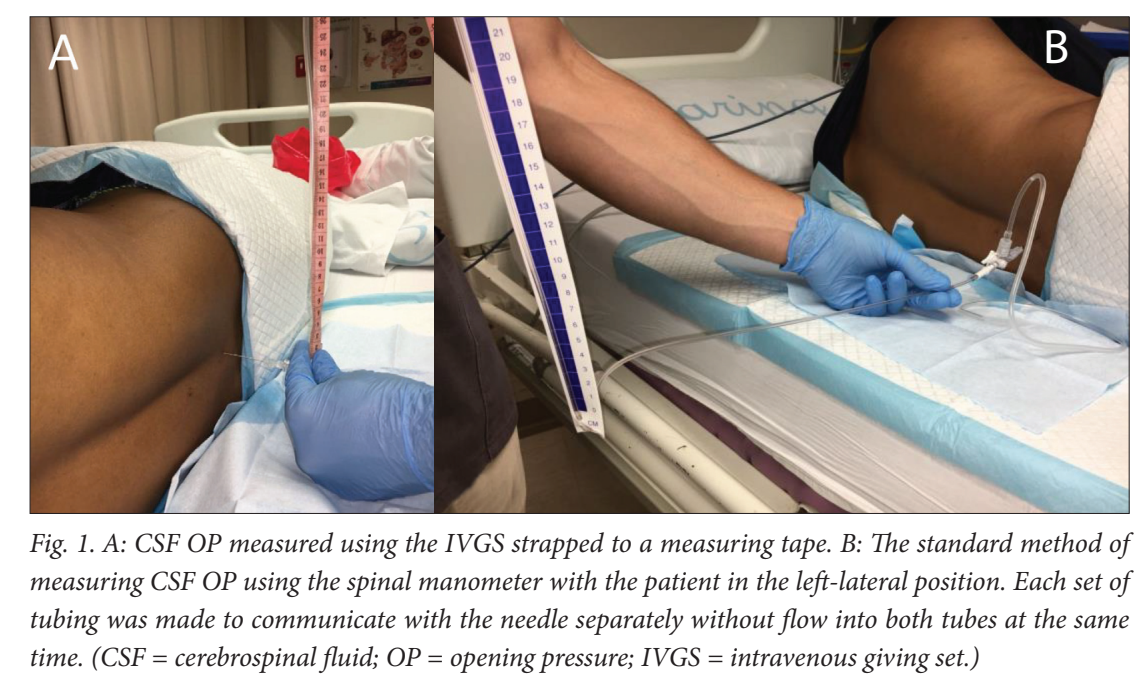

Fig. 1. A: CSF OP measured using the IVGS strapped to a measuring tape. B: The standard method of measuring CSF OP using the spinal manometer with the patient in the left-lateral position. Each set of tubing was made to communicate with the needle separately without flow into both tubes at the same time. $(C S F=$ cerebrospinal fluid; $O P=$ opening pressure; IVGS = intravenous giving set. $)$

or IVGS with small and negligible losses of fluid within the hub of the stopcock. The purpose of using a three-way stopcock was to allow transfer from one measuring instrument to the other with minimal time delay, minimal CSF wastage and no pressure loss. The IVGS tubing was cut to $55 \mathrm{~cm}$ to match the height of the spinal manometer tubing.

The first set of 50 patients had their OPs measured first with the manometer, followed by the IVGS. The second set of 50 patients had their OPs measured first with the IVGS followed by the standard manometer. The rationale behind measuring the $\mathrm{OP}$ by these two methods was to allow for a potential pressure decrease in the second OP measurement from fluid loss into the first tube. It was presumed that greater fluid loss into the $3 \mathrm{~mm}$ bore IVGS would result in a greater pressure decrease measured with the spinal manometer that followed.

Measurement of OP was done by measuring the vertical height in $\mathrm{cm}$ reached by the column of CSF in the IVGS (strapped to a measuring tape) and the spinal manometer (Fig. 1).

\section{Statistical analysis}

Descriptive statistics were used to summarise the data. Continuous variables were summarised by means (standard deviations (SDs)) and categorical data by frequencies and percentages. Pressure measurements were taken by two methods on the same patients. Data were analysed by comparing first all measurements and then measurements by their order of administration. Paired $t$-tests were used to compare the two pressures. Pressures were then dichotomised at $\leq 25 \mathrm{~cm}$ and $>25 \mathrm{~cm}$. McNemar's $\chi^{2}$ test was used to test their agreement. Pearson's correlation was used to measure the strength of the association between the two measure- 
ments, and a linear regression model was used to predict manometer readings based on the IVGS reading. Data were analysed using Stata 13.1 statistical software (StataCorp, USA).

\section{Results}

Table 1 shows a comparison between the groups measured with the manometer first and the IVGS first. There was no significant difference between the groups with regard to demographic parameters and HIV status. The indications for LP, CSF microscopy, culture and tests for chronic infection were also similar. The majority of patients were HIV-positive (70\%), and headache or meningism (40\%) were the commonest reasons for requesting LP. Abnormal biochemical values and pleocytosis were present in $63 \%$ of patients, representing a substantial number of abnormal specimens.

There was a significant difference in the mean CSF OP between the manometer-first and IVGS-first groups (Table 2). Combined group analysis of the 100 patients showed a mean (SD) OP of 22.7 (10.0) $\mathrm{cm}$ CSF with the manometer and $16.2(9.3) \mathrm{cm}$ CSF with the IVGS $(p<0.001)$. Subgroup analysis showed similar findings of lower mean pressure readings with the IVGS (manometer-first group: $23.8 \mathrm{~cm}$ CSF with the manometer v. $16.7 \mathrm{~cm}$ CSF with the IVGS; IVGS-first group: $21.6 \mathrm{~cm}$ CSF with the manometer v. $15.6 \mathrm{~cm}$ CSF with the IVGS). Table 2 shows the SD and $p$-values for both subgroups. There was gross underestimation of the CSF OP by the IVGS, regardless of whether the OP was measured first by the IVGS or the manometer.
The spinal manometer detected 34 cases of elevated CSF pressure ( $>25 \mathrm{~cm}$ CSF) compared with the IVGS, which detected only 11 cases in the combined group analysis (Table 3 ). McNemar's $\chi^{2}$ test showed significant differences in these percentages ( $p<0.001$ for the combined group). The percentage agreement between the manometer and the IVGS in detecting normal and abnormal CSF pressure was $75 \%$. The subgroup analysis showed significant differences in the percentages of elevated CSF pressure in the manometer-first and IVGS-first groups as well ( $p<0.01$, McNemar's $\chi^{2}$ test).

Linear regression of the OP obtained with the manometer and the IVGS showed very good correlation between the two groups (Fig. 2). The correlation coefficient was 0.78 for the whole cohort, 0.82 for the manometer-first group and 0.71 for the IVGS-first group. However, the IVGS consistently underestimated the OP compared with the gold-standard spinal manometer. The linear regression model revealed the following relationship: $M=0.85 V+8.9$, where $M$ was the manometer OP in $\mathrm{cm} \mathrm{CSF}$ and $V$ was the IVGS OP.

\section{Discussion}

Use of an IVGS for measuring CSF OP is common practice in resource-limited settings, but its validity has not been properly tested. The spinal manometer is a tried-and-tested apparatus for CSF pressure measurement, but is not readily available at district and regional hospitals owing to cost and the fact that it is a non-stock item. The latter reason cannot be justified in the absence of validity

\begin{tabular}{|c|c|c|c|}
\hline & Manometer first $(N=50)$ & IVGS first $(N=50)$ & Total $(N=100)$ \\
\hline Age (years), mean (range) & $39.4(20-68)$ & $39.1(18-78)$ & $39.2(18-78)$ \\
\hline Gender female, \% & 48 & 44 & 46 \\
\hline \multicolumn{4}{|l|}{ HIV status, $n(\%)$} \\
\hline Positive & $36(72)$ & $34(68)$ & $70(70)$ \\
\hline Negative & $7(14)$ & $11(22)$ & $18(18)$ \\
\hline Unknown & $7(14)$ & $5(10)$ & $12(12)$ \\
\hline \multicolumn{4}{|l|}{ Indication for LP, $n(\%)$} \\
\hline Headache/meningism & $22(44)$ & $18(36)$ & $40(40)$ \\
\hline Confusion/delirium/ psychosis & $16(32)$ & $17(34)$ & $33(33)$ \\
\hline Recent-onset seizures & $5(10)$ & $6(12)$ & $11(11)$ \\
\hline Therapeutic LP for CCM & $2(4)$ & $3(6)$ & $5(5)$ \\
\hline Other & $5(10)$ & $6(12)$ & $11(11)$ \\
\hline \multicolumn{4}{|l|}{ CSF, $n(\%)$} \\
\hline Abnormal biochemical values & $20(40)$ & $23(46)$ & $43(43)$ \\
\hline Pleocytosis & $9(18)$ & $11(22)$ & $20(20)$ \\
\hline Bloody tap & $9(18)$ & $16(32)$ & $25(25)$ \\
\hline Positive bacterial culture & 0 & 0 & 0 \\
\hline Positive fungal culture & $2(4)$ & $1(2)$ & $3(3)$ \\
\hline Positive CLAT & $4(8)$ & $2(4)$ & $6(6)$ \\
\hline Positive India ink & $3(6)$ & $1(2)$ & $4(4)$ \\
\hline Positive GeneXpert & $0 / 20$ & $0 / 31$ & $0 / 51$ \\
\hline
\end{tabular}

Table 2. Mean CSF OP (cm CSF) in the manometer-first and IVGS-first groups*

\begin{tabular}{|c|c|c|c|c|c|c|c|c|c|c|c|c|}
\hline & \multicolumn{4}{|c|}{ Both groups } & \multicolumn{4}{|c|}{ Manometer first } & \multicolumn{4}{|c|}{ IVGS first } \\
\hline & $N$ & Mean & SD & $p$-value & $N$ & Mean & SD & $p$-value & $N$ & Mean & SD & $p$-value \\
\hline Manometer OP & 100 & 22.7 & 10.0 & & 50 & 23.8 & 11.4 & & 50 & 21.6 & 8.5 & \\
\hline IVGS OP & 100 & 16.2 & 9.3 & & 50 & 16.7 & 11.0 & & 50 & 15.6 & 7.2 & \\
\hline Diff. mano - IVGS & 100 & 6.5 & 6.4 & $<0.001$ & 50 & 7.1 & 6.8 & $<0.001$ & 50 & 6.0 & 6.1 & $<0.001$ \\
\hline
\end{tabular}

$\mathrm{CSF}=$ cerebrospinal fluid; $\mathrm{OP}=$ opening pressure; IVGS = intravenous giving set; $\mathrm{SD}=$ standard deviation, Diff mano - IVGS = difference between the manometer and IVGS pressure reading.

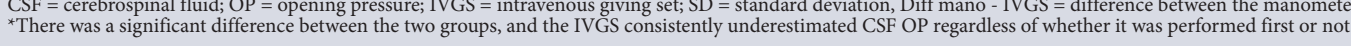


Table 3. Comparison of normal and abnormal CSF OP (cm CSF) between the manometer-first and IVGS-first groups

\begin{tabular}{|c|c|c|c|c|c|c|c|c|c|c|c|c|}
\hline & \multicolumn{4}{|c|}{ Both groups } & \multicolumn{4}{|c|}{ Manometer first } & \multicolumn{4}{|c|}{ IVGS first } \\
\hline & \multicolumn{3}{|c|}{ IVGS OP, $n$} & \multirow[b]{2}{*}{ Abnormal } & \multicolumn{3}{|c|}{ IVGS OP, $n$} & \multirow[b]{2}{*}{ Abnormal } & \multicolumn{3}{|c|}{ IVGS OP, $n$} & \multirow[b]{2}{*}{ Abnormal } \\
\hline & $\leq 25$ & $>25$ & Total & & $\leq 25$ & $>25$ & Total & & $\leq 25$ & $>25$ & Total & \\
\hline Manometer OP, $n$ & & & & Manometer & & & & Manometer & & & & Manometer \\
\hline$\leq 25$ & 65 & 1 & 66 & $34 \%$, IVGS & 31 & 0 & 31 & $38 \%$, IVGS & 34 & 1 & 35 & $30 \%$, IVGS \\
\hline$>25$ & 24 & 10 & 34 & $11 \%(p<0.001$, & 13 & 6 & 19 & $12 \%(p=0.003$ & 11 & 4 & 15 & $10 \%(p=0.004$ \\
\hline Total & 89 & 11 & 100 & $\begin{array}{l}\text { McNemar's } \chi^{2} \\
\text { test) }\end{array}$ & 44 & 6 & 50 & $\begin{array}{l}\text { McNemar's } \chi^{2} \\
\text { test) }\end{array}$ & 45 & 5 & 50 & $\begin{array}{l}\text { McNemar's } \chi^{2} \\
\text { test) }\end{array}$ \\
\hline
\end{tabular}

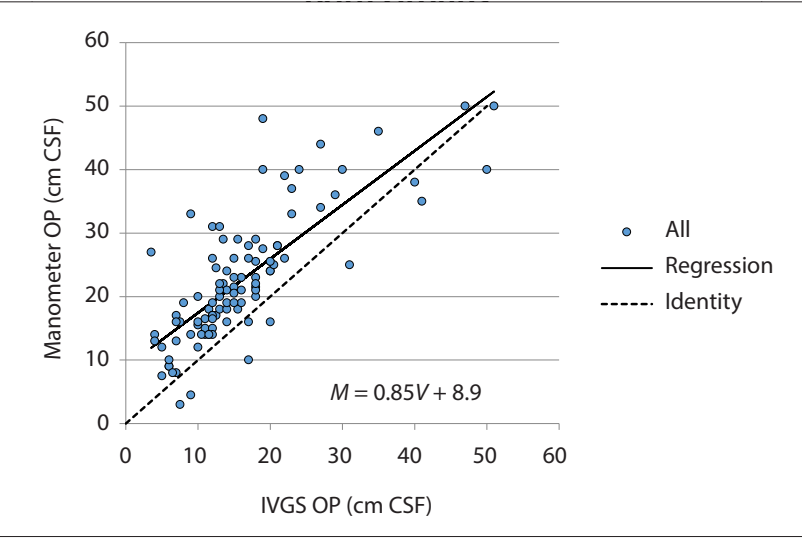

Fig. 2. Linear regression of manometer CSF OP v. IVGS OP showing good correlation between the two despite the underestimation of CSF OP by the IVGS (correlation coefficient $=0.78) . \mathrm{N}=100$ patients, 50 with manometerfirst and 50 with IVGS-first measurements. $(\mathrm{OP}=$ opening pressure; $C S F=$ cerebrospinal fluid; IVGS = intravenous giving set; $\mathrm{M}=$ manometer $O P(\mathrm{~cm}$ CSF), $\mathrm{V}=$ IVGS OP.)

testing of the IVGS for CSF OP measurement. We have addressed this issue in this study. The Tanzanian study ${ }^{[8]}$ showed good correlation between the manometer OP and IVGS OP, but did not show the exact relationship between the two or whether a mathematical correction was required if use of the IVGS to measure CSF pressure became standard practice.

There was no significant difference in demographic parameters, HIV status, CSF biochemical values and microbiology between the manometer-first and IVGS-first groups (Table 1). Inconsistencies recorded in the mean CSF OP were therefore due to the tubing structure of the manometer and IVGS and not to other confounding variables.

We found a significant difference in the mean (SD) CSF OP between the spinal manometer and the IVGS $(22.7$ (10) cm CSF v. 16.2 (9.3) cm CSF; $p<0.001)$. The IVGS consistently underestimated the CSF OP, regardless of whether measurement was performed first with the IVGS or the spinal manometer.

A lowering of the CSF pressure by loss of fluid or compromise of the closed CSF compartment are not plausible reasons for this difference, because regardless of the order in which the spinal manometer was selected for the OP measurement, the manometer readings were always higher than the IVGS readings (Table 2).

Jurin's law of capillary action describes an inverse relationship between the height of capillary action of the fluid within a narrow tube and the tube diameter. ${ }^{[12]}$ It is defined by the equation

$$
h=\frac{2 \gamma \cos \theta}{r \rho g}
$$

where $h$ is the liquid height, $\gamma$ is the surface tension of the liquid, $\theta$ is the contact angle of the liquid on the tube wall, $r$ is the tube radius and $g$ is the gravitational acceleration. Based on Jurin's law, in ideal conditions a $1 \mathrm{~mm}$ bore tube containing water will result in a height of capillary action of $1.5 \mathrm{~cm}$ and a $3 \mathrm{~mm}$ bore tube will result in a height of $0.5 \mathrm{~cm}$. Capillary action is therefore unlikely to result in a mean difference of $6.5 \mathrm{~cm}$ CSF between the $1 \mathrm{~mm}$ bore spinal manometer and the $3 \mathrm{~mm}$ bore IVGS (Table 2). Furthermore, viscosity is unlikely to play a role. Ellis et al. ${ }^{[9]}$ have shown that even with high CSF protein and a pleocytosis, viscosity between CSF and saline are not very different and do not impact appreciably on CSF flow in the clinical setting.

It seems that the $3 \mathrm{~mm}$ bore tube of the IVGS does influence the pressure measurement over that of the $1 \mathrm{~mm}$ bore spinal manometer tube. An equal volume of fluid displaced into the two tubes from the CSF compartment should result in a higher fluid level within the $1 \mathrm{~mm}$ spinal manometer tubing as opposed to the $3 \mathrm{~mm}$ IVGS tubing. With an ideal non-compressible fluid, the level should be 9 times higher in the $1 \mathrm{~mm}$ tube; however, other factors do perhaps play a role, such as higher tube resistance which results in a fluid level lower than expected.

In detecting elevated and normal CSF pressure, the percentage agreement between the manometer and the IVGS was $75 \%$. However, from a clinical perspective, the spinal manometer detected 34 cases of elevated CSF OP as opposed to 11 cases in the IVGS group (Table 3). This difference was statistically significant $(p<0.001$, McNemar's $\chi^{2}$ test) for the combined group analysis. Subgroup analysis also showed significant difference, once again supporting the findings of underestimation of CSF OP by the IVGS (Table 3).

Linear regression revealed good correlation between the manometer OP and the IVGS OP, in spite of the latter being persistently lower. A correlation coefficient of 0.78 for the whole cohort shows a strong correlation between the two. The relationship obtained from regression analysis was $M=0.85 \mathrm{~V}+8.9 \mathrm{in} \mathrm{cm}$ CSF (see Fig. 2). This consistency is helpful in that it suggests that in the resource-limited setting, if use of the IVGS is unavoidable, a fairly reliable manometer estimation of CSF OP can be calculated from the IVGS reading. It is therefore conceivable to estimate CSF OP to an accuracy that is clinically useful by using an IVGS.

The main limitation of this study was that the clinician performing the LPs and measuring the CSF pressure was not blinded to the technique used to obtain the CSF OP. However, the nature of the recording and the different bore sizes did not allow for this blinding.

\section{Conclusions}

In resource-limited settings, linear regression shows that by employing the correction $M=0.85 V+8.9$, a fairly accurate CSF OP can be calculated from the IVGS OP (Fig. 2). A value of $19 \mathrm{~cm} \mathrm{CSF}$ on the IVGS is equivalent to a value of $25 \mathrm{~cm}$ CSF on a manometer, which is the upper limit of normal CSF pressure. A pressure $>19 \mathrm{~cm}$ CSF on the IVGS will therefore indicate elevated CSF pressure. The results of this study have potential to improve the care of patients 
in these settings, especially in the management of cryptococcal- or tuberculosis-induced raised intracranial pressure, which are still common contributors to high mortality in developing countries. ${ }^{[13]}$ However, where qualitative pressure assessment is required in the absence of spinal manometers, Boyles et al. ${ }^{[10]}$ have shown the benefit of the CSF drop-counting method.

Regardless, we still assert that measurement of CSF OP should be performed by standard spinal manometry, a tried-and-tested method of measuring CSF OP. The practice of using the IVGS to measure CSF OP should be discouraged, especially if no correction of the result is made. The current situation in SA is suboptimal and must be addressed at a national level so that manometers are available at all levels of care. The spinal manometer must be classified as an essential stock item that is readily available when required, and negotiation for better tender prices should be initiated at national level.

Acknowledgements. The authors are grateful to all patients who participated in this study and to the nursing staff who assisted during the LPs.

Author contributions. Conception and design of the work: TAM, AM; data collection: TM; data analysis and interpretation: TM, AM, CC; drafting the article: TAM, AM; critical revision of the article: AM; final approval of the version to be published: TM, AM, CC.

Funding. None.

Conflicts of interest. None.
1. Graybill JR, Sobel J, Saag M, et al. Diagnosis and management of increased intracranial pressure in patients with AIDS and cryptococcal meningitis: The NIAID Mycoses Study Group and AIDS in patients with AIDS and cryptococcal meningitis: The NIAID Mycoses Study Group and A

2. Gambarin KJ, Hamill RJ. Management of increased intracranial pressure in cryptococcal meningitis. Gambarin KJ, Hamill RJ. Management of increased intracranial pressure in crypto
Curr Infect Dis Rep 2002;4(4):332-338. https://doi.org/10.1007/s11908-002-0026-y

Curr Infect Dis Rep 2002;4(4):332-338. https://doi.org/10.1007/s11908-002-0026-y
Robertson EJ, Najiuka G, Rolfes MA, et al. Cryptococcus neoformans ex vivo capsule size is associated . Robertson EJ, Najjuka G, Rolfes MA, et al. Cryptococcus neoformans ex vivo capsule size is associated
with intracranial pressure and host immune response in HIV-associated cryptococcal meningitis. with intracranial pressure and host immune response in HIV-asso
Infect Dis 2014;209(1):74-82. https://doi.org/10.1093/infdis/jit435

4. Fessler RD, Sobel J, Guyot L, et al. Management of elevated intracranial pressure in patients with cryptococcal meningitis. J Acquir Immune Defic Syndr Hum Retrovirol 1998;17(2):137-142.

5. Bicanic T, Brouwer AE, Meintjes G, et al. Relationship of cerebrospinal fluid pressure, fungal burden and outcome in patients with cryptococcal meningitis undergoing serial lumbar punctures. AIDS 2009;23(6):701-706. https://doi.org/10.1097/QAD.0b013e32832605fe

6. Whiteley W, Al-Shahi R, Warlow CP, Zeidler M, Lueck CJ. CSF opening pressure: Reference interval and the effect of body mass index. Neurology 2006;67(9):1690-1691. https://doi.org/10.1212/01. wnl.0000242704.60275.e9

7. Lindvall P, Ahlm C, Ericsson M, Gothefors L, Naredi S, Koskinen LD. Reducing intracranial pressure may increase survival among patients with bacterial meningitis. Clin Infect Dis 2004;38(3):384-390. may increase survival among p

8. Meda J, Kalluvya S, Downs JA, et al. Cryptococcal meningitis management in Tanzania with strict schedule of serial lumber punctures using intravenous tubing sets: An operational research study. Acquir Immune Defic Syndr 2014;66(2)e31-e36. https://doi.org/10.1097/QAI.0000000000000147

9. Ellis 3rd RW, Strauss LC, Wiley JM, Killmond TM, Ellis Jr. RW. A simple method of estimating cerebrospinal fluid pressure during lumbar puncture. Pediatrics 1992;89(5 Pt 1):895-897.

10. Boyles TH, Gatley E, Wasserman S, Meintjes G. Flow rate of cerebrospinal fluid through a spinal needle can accurately predict intracranial pressure in cryptococcal meningitis. J Acquir Immune Defic Syndr 2017;74(3):e64-e66. https://doi.org/10.1097/QAI.0000000000001183

1. Pfeiffer BM, Kampmann C, Wirth S, Trübel H. Measuring opening pressure in lumbar puncture - a new method. Biomed Tech (Berl) 2003;48(4):86-89.

12. Rodríguez-Valverde MA, Miranda MT. Derivation of Jurin’s law revisited. Eur J Phys 2011;32(1):49-54. https://doi.org/10.1088/0143-0807/32/1/005

13. Rolfes MA, Hullsiek KH, Rhein J, et al. The effect of therapeutic lumbar punctures on acute mortality from cryptococcal meningitis. Clin Infect Dis 2014;59(11):1607-1614. https://doi.org/10.1093/cid/ ciu596

Accepted 18 April 2018. 